\title{
Differential gene expression profiling of primary cutaneous melanoma and sentinel lymph node metastases
}

\author{
Stephen S Koh ${ }^{1}$, Jia-Perng J Wei ${ }^{1}$, Xinmin $\mathrm{Li}^{1}$, Rong R Huang ${ }^{1}$, Ngan B Doan ${ }^{1}$, \\ Richard A Scolyer ${ }^{2,3,4}$, Alistair J Cochran ${ }^{1,5,6}$ and Scott W Binder ${ }^{1}$
}

${ }^{1}$ Department of Pathology and Laboratory Medicine, University of California Los Angeles/David Geffen School of Medicine at UCLA, Los Angeles, CA, USA; ${ }^{2}$ Department of Tissue Pathology and Diagnostic Oncology, Royal Prince Alfred Hospital, Sydney, NSW, Australia; ${ }^{3}$ Melanoma Institute Australia, Sydney, NSW, Australia; ${ }^{4}$ Discipline of Pathology, Sydney Medical School, University of Sydney, Sydney, NSW, Australia; ${ }^{5}$ Department of Surgery, Jonsson Comprehensive Cancer Center, UCLA, Los Angeles, CA, USA and ${ }^{6} J o h n$ Wayne Cancer Institute, Santa Monica, CA, USA

\begin{abstract}
Limited understanding of molecular mechanisms of metastasis in melanoma contributes to the absence of effective treatments. Increased knowledge of alterations in genes that underpin critical molecular events that lead to metastasis is essential. We have investigated the gene expression profiles of primary melanomas and melanoma metastases in sentinel lymph nodes. A total of 19 samples (10 primary melanomas and 9 sentinel lymph node metastases) were evaluated. Melanoma cells were dissected from tissue blocks. Total mRNA was isolated, amplified, and labeled using an Ambion Recover All Total Nucleic Acid Isolation kit, Nu-GEN WTOvation formalin-fixed, paraffin-embedded RNA Amplification System, and FL-Ovation cDNA Biotin Module V2, respectively. Samples were hybridized to the Affymetrix Gene Chip Human U133 Plus 2.0 Array. Data were analyzed using Partek Genomics Suite Version 6.4. Genes selected showed $\geq 2$-fold difference in expression and $P<5.00 E-2$. Validation studies used standard immunohistochemical assays. Hierarchical clustering disclosed two distinct groups: 10 primary melanomas and 9 sentinel lymph node metastases. Gene expression analysis identified $\mathbf{5 7 6}$ genes that showed significant differential expression. Most differences reflected decreased gene expression in metastases relative to primaries. Reduced gene expression in primaries was less frequent and less dramatic. Genes significantly increased or decreased in sentinel lymph node metastases were active in cell adhesion/structural integrity, tumor suppression, cell cycle regulation, and apoptosis. Validation studies indicate that MAGEC1 (melanoma antigen family C1) and FCRL1 (Fc receptor-like 1) are involved in melanoma progression. There are striking differential gene expression patterns between primary and nodally metastatic melanomas. Similar findings were seen with autologous paired primary melanomas and sentinel lymph node metastases, supporting involvement of these gene alterations in evolution of metastases. With further study, it may be possible to determine the exact sequence of molecular events that underlie melanoma metastases. Modern Pathology (2012) 25, 828-837; doi:10.1038/modpathol.2012.32; published online 9 March 2012
\end{abstract}

Keywords: DNA microarray; formalin-fixed, paraffin-embedded tissue; gene expression; melanoma; sentinel lymph node

Correspondence: Dr SW Binder, MD, Department of Pathology and Laboratory Medicine, University of California Los Angeles Medical Center/David Geffen School of Medicine at UCLA, 10833 Le Conte Avenue, Box 951732, 13-186 CHS, Los Angeles, CA 90095-1732, USA.

E-mail: sbinder@mednet.ucla.edu

Received 27 May 2011; revised 27 September 2011; accepted 27 September 2011; published online 9 March 2012
Early detection and complete surgical excision of the primary tumor offer the best chance of cure for melanoma patients. Sentinel lymph node biopsy is recommended for clinically localized primary melanomas $\geq 1 \mathrm{~mm}$ thick, or thinner tumors with adverse prognostic features, such as mitotic activity $\left(\geq 1\right.$ per $\left.\mathrm{mm}^{2}\right)$. Complete lymph node dissection is recommended for patients with a tumorpositive sentinel lymph node. Long-term follow-up 
highlights the importance of lymph-node staging and its accuracy as a prognostic indicator. The 2010 American Joint Committee on Cancer database contained 3307 stage III patients with 5-year survival rates of 78,59 , and $40 \%$ for stages IIIA, IIIB, and IIIC, respectively, ${ }^{1}$ indicating that survival decreases as nodal tumor burden increases. The Multicenter Selective Lymphadenectomy Trial I demonstrated that patients with a tumor-positive sentinel lymph node had decreased 5-year diseasefree survival rates relative to sentinel lymph-nodenegative patients. Sentinel lymph node status is the most important prognostic factor for melanoma patients with clinically localized disease. ${ }^{2}$ The sentinel lymph node is the initial site of metastasis for most melanoma patients, but the mechanisms, which permit melanoma cells to invade and traverse the lymphatic system, and survive and proliferate in lymph nodes, are poorly understood. Molecular biology techniques, such as DNA microarray expression profiling and associated bioinformatics approaches, promise advances in our understanding of these critical issues.

Functional genomics (transcriptomics) permit simultaneous analysis of the expression of thousands of genes, critically advanced with the development of DNA microarray systems in the mid 1990's. ${ }^{3,4}$ These techniques have been used to identify disease-associated biomarkers, create genebased tumor classifications, distinguish tumor subclasses, and predict outcomes and responses to chemotherapy. ${ }^{5}$ The DNA microarray technique has advanced our understanding of breast cancer, lung cancer, rhabdomyosarcoma, glioblastoma, and melanoma. In 2000, Bittner et $a l^{6}$ reported the gene expression profiles of melanoma cells in fresh frozen tissues and cell cultures, showing differential gene expression in a subset of melanomas with aggressive metastatic features. Others, using cell lines or tissue samples, have studied DNA expression profiling of normal melanocytes, nevi, primary melanomas, and regional and distant metastases. ${ }^{7-19}$ Conway et $a l^{19}$ used the cDNA-mediated annealing, selection, extension, and ligation assay to evaluate formalin-fixed, paraffin-embedded archival primary melanoma specimens, and reported osteopontin overexpression as a potential prognostic biomarker. Rangel et $a l,{ }^{20}$ using immunohistochemistry, had previously shown that overexpression of osteopontin, correlated with the likelihood of sentinel lymph node metastasis.

In this study, we used the Affymetrix Gene Chip Human U133 Plus 2.0 Array to conduct a 'wholegenome' expression profiling of primary melanomas and melanoma metastases in sentinel lymph nodes. In contrast to prior studies that utilized limited DNA microarray chip systems, the technology used in this study screens transcripts that closely approach representation of the whole-human genome. The analyses were designed to gain further understanding of the mechanisms of melanoma metastasis, and in particular, of the genes responsible for the capacity of melanoma to metastasize to lymph nodes.

\section{Materials and methods}

\section{Case Selection and Sample Collection}

Formalin-fixed, paraffin-embedded tissue samples, up to 3 years old, were obtained from the resources of the UCLA Department of Pathology and Laboratory Medicine, and the Department of Tissue Pathology and Diagnostic Oncology, Royal Prince Alfred Hospital, Sydney. Collection of samples and study protocols were approved by the institutional review boards at the contributing institutions: University of California Los Angeles (IRB number 05-10-038-11) and Sydney South West Area Health Service Ethics Review Committee (RPAH Zone).

Tissue blocks of primary melanomas $(1.9-6.0 \mathrm{~mm}$ in thickness) and sentinel lymph node metastases (2-24 $\mathrm{mm}$ in greatest diameter/aggregate diameter) were selected, taking into consideration the ease with which dissection could be performed to provide tumor tissue relatively free of contaminating nodal and skin components. The tumor samples utilized for analysis were comprised of not less than $80 \%$ tumor cells. Highly pigmented specimens were avoided due to previously published technical issues that reported low RNA yield in the presence of abundant melanin. ${ }^{19} \mathrm{~A}$ total of 22 samples were screened; however, only 19 samples were used, 3 being excluded on the basis of poor RNA quality. In all, 10 primary melanomas and 9 sentinel lymph node metastases were evaluated, including 4 autologous pairs of primary melanoma and sentinel lymph node metastasis (from 4 patients). The remaining 11 unpaired samples were 6 primaries and 5 sentinel lymph nodes from 11 patients.

The melanoma specimens were procured, on average, from surgeries performed 18 months before our evaluation (range 6-36 months). Hematoxylin and eosin-stained sections of each case were independently reviewed by 3-4 dermatopathologists during selection to verify the diagnosis and quantitate the tumor content in the sample. Tumor tissue from 13 large lesions was dissected from the tissue blocks. Nine moderately sized lesions were sectioned at $20-\mu \mathrm{m}$ thickness and placed on uncharged slides (10-20 sections). After defining the target tumor mass on hematoxylin and eosin-stained slides, the tumor tissue was carefully dissected manually or using a laser dissection microscope, depending on the size of the tumor mass and the distribution of the tumor. Purity of samples (percentage of tumor cells in the sample) was at least $80 \%$ in all cases (range $80-90 \%$ ).

\section{RNA Isolation and Microarray Hybridization}

All RNA extraction and microarray experiments were performed at the UCLA Department of Pathology 
Clinical Microarray Core Laboratory. Total RNA was isolated using the Ambion RecoverAll (Applied Biosystems/Ambion, Austin, TX, USA) kit according to the manufacturer's instructions. RNA Integrity was evaluated using an Agilent 2100 Bioanalyzer (Agilent Technologies, Palo Alto, CA, USA) and purity/ concentration was determined using a NanoDrop 8000 (NanoDrop Products, Wilmington, DE, USA). Microarray targets were prepared using NuGEN WTOvation formalin-fixed, paraffin-embedded RNA Amplification System and FL-Ovation cDNA Biotin Module V2 (NuGEN Technologies, San Carlos, CA, USA) and then hybridized to the Affymetrix GeneChip U133plus 2.0 Array (Affymetrix, Santa Clara, CA, USA), all according to manufacturers' instructions. The arrays were washed and stained with streptavidin phycoerythrin in Affymetrix Fluidics Station 450 using the Affymetrix GeneChip protocol, and then scanned using an Affymetrix GeneChip Scanner 3000.

\section{Data Analysis}

The acquisition and initial quantification of array images were conducted using the AGCC software (Affymetrix). The subsequent data analyses were performed using Partek Genomics Suite Version 6.4 (Partek, St Louis, MO, USA). Differentially expressed genes were selected at $\geq 2$-fold difference between primary and metastases, and $P<5.00 \mathrm{E}-2$. Cluster analyses and principal component analysis were conducted with Partek default settings. Biofunctional analysis was performed using Ingenuity Pathways Analysis software Version 7.6 (Ingenuity Systems, Redwood City, CA, USA).

\section{Validation by Immunohistochemistry}

Immunohistochemistry was performed using antibodies to protein products of the genes listed below. Paraffin-embedded sections were cut at $4-\mu \mathrm{m}$ thickness deparaffinized with xylene, and rehydrated through graded ethanol. Endogenous peroxidase activity was blocked with $3 \%$ hydrogen peroxide in methanol for $10 \mathrm{~min}$. Heat-induced antigen retrieval was carried out for most sections in either $0.001 \mathrm{M}$ EDTA buffer, $\mathrm{pH}=8.00$, or $0.001 \mathrm{M}$ citrate, $\mathrm{pH}=6.00$, using a vegetable steamer at $95{ }^{\circ} \mathrm{C}$ for $25 \mathrm{~min}$. Proteolytic-induced epitope retrieval with proteinase K (Dako, S3020, Carpinteria, CA, USA) at $37^{\circ} \mathrm{C}$ for $10 \mathrm{~min}$ was used for S100A8. After being washed with PBST, the sections were incubated with polyclonal goat anti-SFN (stratifin; Abnova, Walnut, CA, USA), monoclonal rabbit anti-cytokeratin 6 (Epitomics, Burlingame, CA, USA), monoclonal mouse anti-MAGE-C1 (Santa Cruz Biotechnology, Santa Cruz, CA, USA), polyclonal rabbit anti-FCRL1 (FC receptor-like 1; Sigma Aldrich, St Louis, MO, USA), monoclonal mouse antiMaspin (aka SERPINB5; BD Bioscience, Cat. number
554292), monoclonal mouse anti-MMP1 (EMD, Oncogene Research Products, Cat. number IM35L), monoclonal mouse anti-CD20 (aka MS4A1; DakoCytomation, Cat. number M0755), and monoclonal mouse anti-S100A8/Anti-Migration Inhibitory Factor Related Proteins 8 and 14, clone AHN-17 I (Millipore, Cat. number MAB1789) at 1:50, 1:50, 1:50, 1:10, $1: 200,1: 200,1: 1000$, and 1:200 dilutions, respectively, for $16 \mathrm{~h}$ at $4{ }^{\circ} \mathrm{C}$ or $2 \mathrm{~h}$ at 90 , or $45 \mathrm{~min}$ at room temperature. Following overnight incubation, sections stained for SFN were incubated with secondary rabbit anti-goat immunoglobulins/biotinylated (Dako, E0466) at 1:200 for $30 \mathrm{~min}$. For all sections, the signal was detected using either Labelled Polymer Horseradish Peroxidase anti-mouse or anti-rabbit EnVision system (Dako) and visualized with the diaminobenzidine reaction. The sections were counterstained with hematoxylin.

\section{Results}

Characteristics of Patients and Tumors Evaluated by Gene Expression Profiling

Table 1 summarizes the clinical and pathological characteristics of the patients and tumors analyzed in the study. Detailed clinical and pathology data were available for the 15 patients whose primary and metastatic melanomas provided the 19 formalin-fixed, paraffin-embedded tissue blocks used for analysis. Four patients (cases 12-15) provided paired samples of primary melanoma and sentinel lymph node metastases for analysis. The other 11 patients provided either a primary melanoma (cases 1-6) or a sentinel lymph node metastasis (cases 711). All primary melanomas had a bulky vertical growth phase component. Two extended to the papillary-reticular interface (Clark level III), four extended into the reticular dermis (Clark level IV), and four invaded the subcutaneous fat (Clark level V). The mean Breslow thickness was $3.6 \mathrm{~mm}$ (range: $1.9-5.3 \mathrm{~mm}$ ). Four of the unmatched primary melanomas cases (cases 1-3,5) had no evidence of nodal metastasis. Two of the unmatched primary melanoma (cases 4 and 6) had regional nodal metastasis. Case 1 had distant metastasis to the lung. No distant metastases were identified in cases $2-6$. The remaining nine patients with a tumorpositive sentinel lymph node (cases 7-15) had a completion lymphadenectomy and six of these cases had non-sentinel lymph node metastases. Eight of these nine latter cases had distant metastasis.

\section{Hierarchical Clustering and Principal Component Analysis Identifies Distinctive Molecular Phenotypes in Primary Melanoma And Sentinel Lymph Node Metastases}

DNA microarray expression profiling demonstrated significant differences in gene expression between primary melanomas and metastases in sentinel 


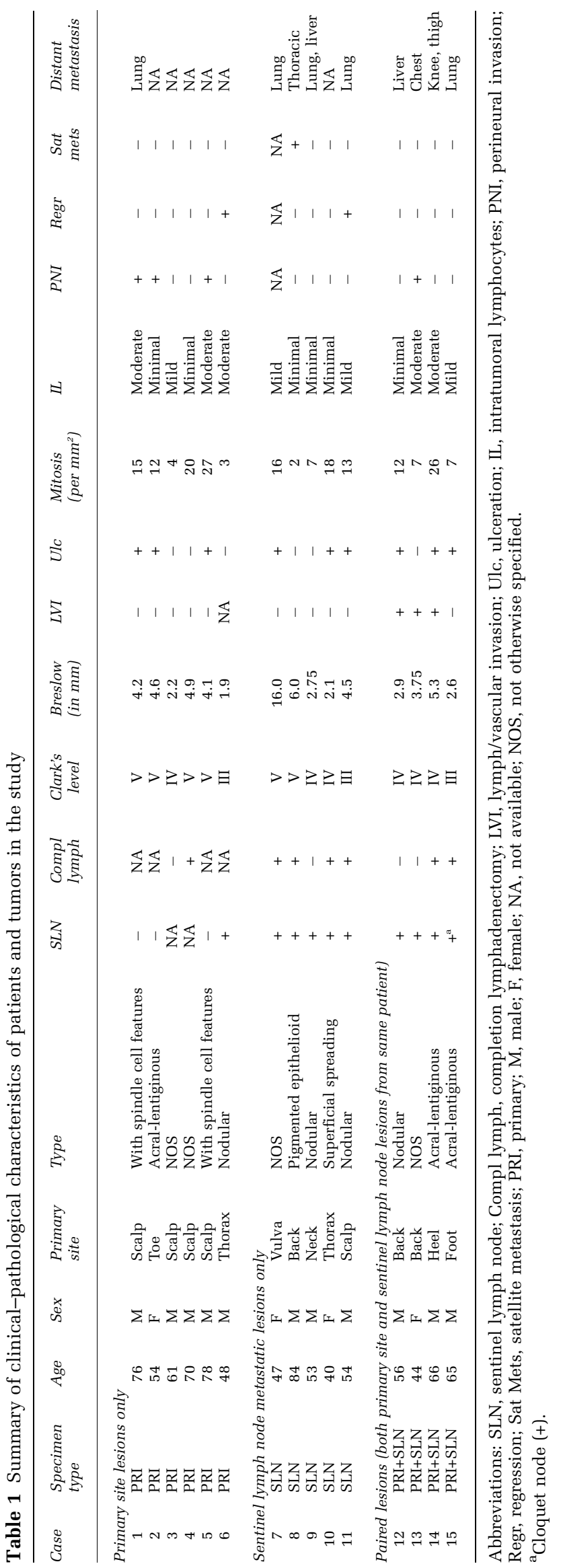

nodes. A gene was accepted as differentially expressed if there was $P$-value of $<5.00 \mathrm{E}-2$ and two-fold or greater difference in its expression between the study groups. This segregated 576 statistically significant probes that represented multiple genes. A hierarchical clustering map was generated utilizing the significant differentially expressed probes/genes of interest. The heat map revealed two distinctive patterns that closely correlated with the two study groups. Primary melanomas are depicted in the lower half of the hierarchical tree in purple and sentinel lymph node metastases in the upper half of the hierarchical tree in green. Genes that showed increased expression are shown in red and genes that showed decreased expression in blue in Figure 1a. The 402 probes/genes in the larger area of the upper right quadrant of the cluster map were genes that were decreased in sentinel lymph node samples relative to the primary melanoma samples shown in the lower right quadrant. The 174 probes/genes in the smaller left upper quadrant were genes that were increased in sentinel lymph node samples relative to primary melanoma samples in the lower left quadrant.

To better visualize the large data sets obtained from these DNA expression microarray studies, principal component analysis was performed. Figure 1b illustrates a three-dimensional scatter plot derived from the gene list utilized in constructing the hierarchical clustering map in Figure 1a. This approach determines the pattern of samples that form distinct groups. In this case, primary melanomas (purple) were easily distinguishable from sentinel lymph node metastases (green). There were no significant outliers.

\section{Gene expression Profiling Identified Genes that were Expressed at Significantly Different Frequency in Primary Melanomas and Sentinel Nodal Metastases}

The Affymetrix Gene Chip Human U133 Plus 2.0 Array used in this study includes more than 54000 probe sets that can analyze the relative expression of more than 47000 transcripts, offering a virtually comprehensive expression analysis of the entire genome. A working list of 576 candidate probe sets was developed on the basis of the statistical significance $(P<5.00 \mathrm{E}-2)$ of the difference in their expression of the two study groups and an at least two-fold change in gene frequency $(\geq 2)$ between primary and metastatic melanomas. A total of 174 of these probe sets were significantly overexpressed in sentinel lymph node metastases relative to primary melanomas. The remaining 402 probe sets were significantly underexpressed in sentinel lymph node metastases relative to primary melanomas.

The 174 probe sets that were overexpressed in sentinel lymph node samples were sorted in descending order of fold change (greatest to least). The 35 probe sets with the greatest overexpression are listed in Table 2a. Fold difference of expression ranged from 

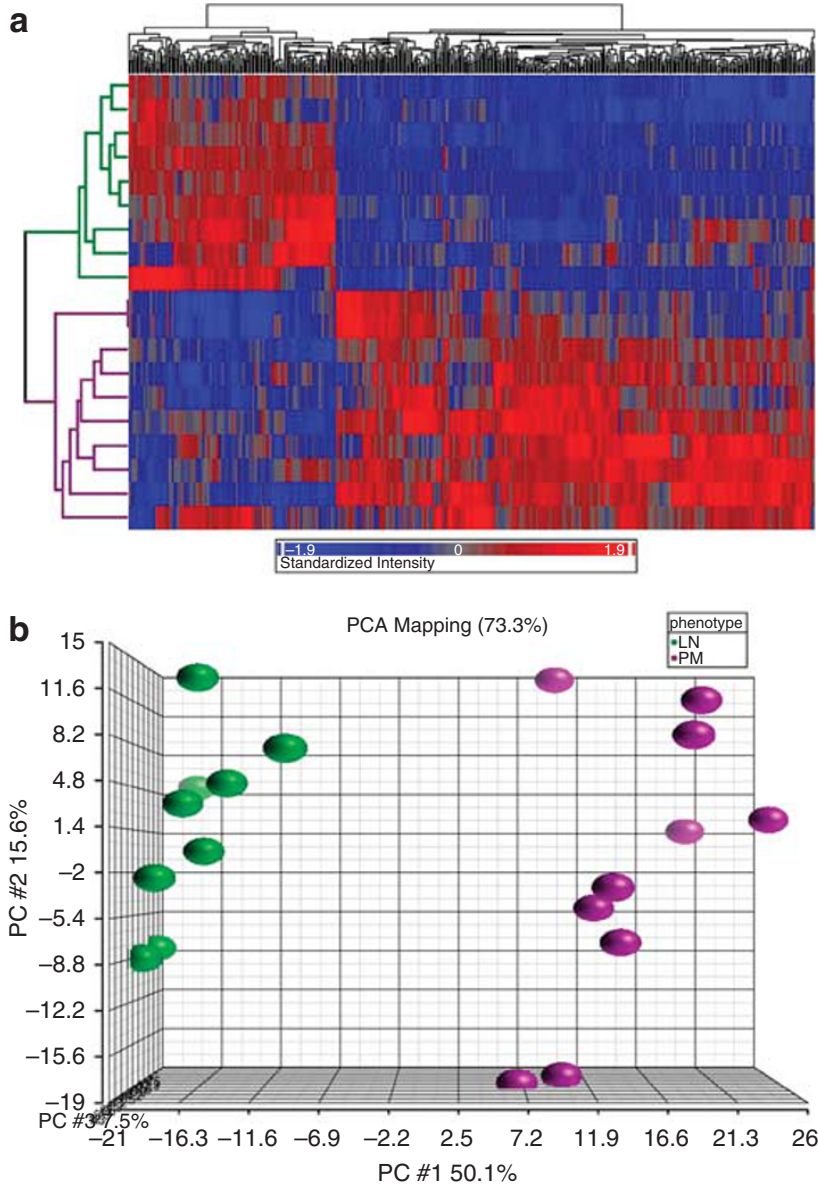

Figure 1 Analysis of all 19 samples resulted in two relatively distinct patterns distinguishing the 9 sentinel lymph node melanoma metastases from the remaining 10 primary melanomas. (a) Hierarchical clustering of primary melanoma vs sentinel lymph node melanoma metastases. Each row represents each sample tested and each column represents a single probe. On the hierarchical tree at the left side of the diagram, the upper half (green) indicates the sentinel lymph node melanoma metastases and the lower half (purple) indicates the primary melanomas. Relative gene expression is color represented: red is the higherlevel expression, blue is the lower-level expression, and grey is no change. (b) Principal component analysis of primary melanomas vs sentinel lymph node melanoma metastases. Analysis of 19 samples resulted in two relatively distinct components of nine sentinel lymph node melanoma metastases (green) and 10 primary melanomas (purple).

7.55239 to 2.79073 and $P$-values from $2.19 \mathrm{E}-02$ to $9.91 \mathrm{E}-06$. Some genes and gene family members identified, such as MAGE, FCRL1, MS4A1, and XIST, had multiple probe sets. Probe set ID's for the Affymetrix array are listed in the left column. Genes that are currently uncharacterized are listed as '- ' in the gene symbol column and with a public National Center for Biotechnology Information gene bank accession identification in the gene name column. Some of the uncharacterized genes are also labeled as 'hypothetical protein' in the gene name column. Although some genes with great fold difference in expression are currently uncharacterized, many, such as MAGEC1 (melanoma antigen family C1) and
FCRL1, have been identified and information concerning their biological roles is available.

The 402 probe sets with lower expression in the sentinel lymph node metastases, were also sorted by greatest fold change and significance. The 35 probe sets with the greatest fold difference were identified and are listed in Figure 2b. Fold difference ranged from 75.9615 to 7.08074 and $P$-values from $8.74 \mathrm{E}-04$ to $1.02 \mathrm{E}-10$. Multiple probe sets were identified for the gene $S F N$. Multiple subtypes of the $S 100$ family had significant fold difference in expression, including S100A8, S100A7, and S100A2. The KRT, DSC, SERPINB, and SPRR also had multiple subtypes. Biofunctional analysis showed that genes that were 'downregulated' in sentinel lymph node metastases were mostly associated with structural cellular proteins (keratins, desmoglein, and gap junction proteins). Others were tumor suppressor genes, such as stratifin, serpin peptidase inhibitor, and chloride channel accessory.

\section{Analysis of Autologous Primary Melanomas and Sentinel Lymph Node Metastases}

Hierarchical clustering of autologous primary melanomas and melanomas metastatic to the sentinel lymph node was undertaken, separately from the 11 unpaired samples. These four primary melanoma samples with their autologous sentinel lymph node metastasis generated the differential expression patterns illustrated in Figure 2a. There was a distinct separation between samples from primary and metastatic melanomas that closely resembled the pattern of the heat map generated from all 19 samples (Figure 1a). Principal component analysis (Figure 2b) showed a clear separation between primaries and sentinel lymph node metastases without outliers, a pattern identical to that seen in our analysis of all samples (Figure 1b). Hierarchical clustering of the 11 unpaired samples resulted in a similar differential expression pattern to that seen with the total 19 samples and the 8 paired samples (data not shown).

\section{Expression Array Data Validation by Immunohistochemistry}

Validation studies were performed using standard immunohistochemistry. From the gene lists (Table 2a and b), representative markers (MAGEC1, FCRL1, MS4A1, SFN, KRT6, SERPINB5, MMP1, S100A8) were selected and assayed on sections from one primary melanoma (Table 1, case 5, Figure 3a) and one sentinel lymph node metastasis (Table 1, case 7 , Figure $3 b$ ). Selection of cases 5 and 7 was based on the availability of sufficient material for testing in the block remnants. From the list of genes that showed relatively 'increased expression' in sentinel lymph node metastases (Table 2a), immunohistochemistry targeted to MAGEC1 and FCRL1 gene 
Table 2 Representative genes with greatest fold differences

\begin{tabular}{ll}
\hline Probeset ID Gene symbol $\quad$ Gene name & Fold difference \\
(increased in SLN)
\end{tabular}

(a) 'Upregulated' gene expression in association with metastasis. Genes with increased expression in SLN melanoma metastases vs primary melanomas

\begin{tabular}{|c|c|c|c|c|}
\hline 235446_at & - & AW856618 NCBI & 7.55239 & $2.26 \mathrm{E}-05$ \\
\hline 243968_x_at & FCRL1 & Fc receptor-like 1 & 5.5191 & $2.09 \mathrm{E}-04$ \\
\hline 235982_at & FCRL1 & Fc receptor-like 1 & 5.36621 & $3.02 \mathrm{E}-05$ \\
\hline 214218_s_at & XIST & $\mathrm{X}$ (inactive)-specific transcript (non-protein coding) & 5.36364 & $1.05 \mathrm{E}-03$ \\
\hline 224590_at & XIST & $\mathrm{X}$ (inactive)-specific transcript (non-protein coding) & 5.02399 & $3.94 \mathrm{E}-04$ \\
\hline 224588_at & XIST & X (inactive)-specific transcript (non-protein coding) & 4.86516 & $9.30 \mathrm{E}-04$ \\
\hline 231592_at & TSIX & XIST anti-sense RNA (non-protein coding) & 4.78334 & $7.59 \mathrm{E}-05$ \\
\hline 206609_at & MAGEC1 & Melanoma antigen family C, 1 & 4.72449 & $5.82 \mathrm{E}-04$ \\
\hline 209942_x_at & MAGEA3 & Melanoma antigen family A, 3 & 4.49082 & $1.76 \mathrm{E}-03$ \\
\hline 214612_x_at & $M A G E A 6$ & Melanoma antigen family A, 6 & 4.27124 & $1.30 \mathrm{E}-03$ \\
\hline 210467_x_at & MAGEA12 & Melanoma antigen family A, 12 & 4.2667 & $1.84 \mathrm{E}-03$ \\
\hline 243712_at & XIST & $\mathrm{X}$ (inactive)-specific transcript (non-protein coding) & 4.26661 & $2.86 \mathrm{E}-04$ \\
\hline 227671_at & XIST & $\mathrm{X}$ (inactive)-specific transcript (non-protein coding) & 4.23126 & $1.48 \mathrm{E}-04$ \\
\hline 1554301_at & $L O C 645591$ & Hypothetical protein LOC645591 & 4.23053 & $1.51 \mathrm{E}-02$ \\
\hline 241535_at & LOC100292909 & Hypothetical protein LOC100292909 & 4.14673 & $4.45 \mathrm{E}-03$ \\
\hline 210356_x_at & MS4A1 & Membrane-spanning 4-domains, subfamily A, member 1 & 3.68644 & $4.49 \mathrm{E}-03$ \\
\hline 217418_x_at & $M S 4 A 1$ & Membrane-spanning 4-domains, subfamily A, member 1 & 3.61983 & $2.92 \mathrm{E}-03$ \\
\hline 240366_at & LOC100129562 & Hypothetical protein LOC100129562 & 3.4838 & $2.19 \mathrm{E}-02$ \\
\hline 1568609_s_at & $F A M 91 A 2^{\mathrm{a}}$ & Family with sequence similarity 91, member A2 /// hypothetical FLJ39739 /// hypo & 3.47266 & $1.59 \mathrm{E}-03$ \\
\hline 221728_x_at & XIST & X (inactive)-specific transcript (non-protein coding) & 3.3718 & $1.18 \mathrm{E}-03$ \\
\hline 229872_s_at & LOC100132999 & Hypothetical protein LOC100132999 & 3.36217 & $3.21 \mathrm{E}-03$ \\
\hline 221969_at & - & BF510692 NCBI & 3.26099 & $1.56 \mathrm{E}-03$ \\
\hline 1554302_s_at & LOC645591 & Hypothetical protein LOC645591 & 3.17599 & $2.10 \mathrm{E}-02$ \\
\hline 220062_s_at & MAGEC2 & Melanoma antigen family C, 2 & 3.13847 & $2.52 \mathrm{E}-04$ \\
\hline 210448_s_at & P2RX5 & Purinergic receptor P2X, ligand-gated ion channel, 5 & 3.07721 & $1.25 \mathrm{E}-03$ \\
\hline 224589_at & XIST & X (inactive)-specific transcript (non-protein coding) & 3.07169 & $2.64 \mathrm{E}-03$ \\
\hline 231418_at & - & AI808597 NCBI & 3.02583 & $1.93 \mathrm{E}-02$ \\
\hline 209685_s_at & $P R K C B$ & Protein kinase $\mathrm{C}$, beta & 2.97829 & $9.91 \mathrm{E}-06$ \\
\hline 219667_s_at & $B A N K 1$ & B-cell scaffold protein with ankyrin repeats 1 & 2.95487 & $1.36 \mathrm{E}-02$ \\
\hline 226344_at & $Z M A T 1$ & Zinc finger, matrin type 1 & 2.94824 & $5.88 \mathrm{E}-03$ \\
\hline 240050_s_at & - & BF751607 NCBI & 2.91584 & $7.04 \mathrm{E}-04$ \\
\hline 230983_at & FAM129C & Family with sequence similarity 129 , member C & 2.88593 & $2.12 \mathrm{E}-04$ \\
\hline 1562294_x_at & ANKRD30B & Ankyrin repeat domain 30B & 2.81943 & $9.92 \mathrm{E}-03$ \\
\hline 244061_at & - & AI510829 NCBI & 2.79343 & $7.33 \mathrm{E}-04$ \\
\hline 229073_at & PRTG & Protogenin homolog (Gallus gallus) & 2.79073 & $2.94 \mathrm{E}-03$ \\
\hline
\end{tabular}

(b) 'Downregulated' gene expression in association with metastasis. Genes with decreased expression in SLN node melanoma metastases vs primary melanomas

\begin{tabular}{|c|c|c|c|c|}
\hline 209125_at & KRT6A & Keratin 6A & 75.9615 & $1.59 \mathrm{E}-08$ \\
\hline 33323_r_at & SFN & Stratifin & 74.3948 & $3.30 \mathrm{E}-10$ \\
\hline 213680_at & KRT6B & Keratin 6B & 66.036 & $1.88 \mathrm{E}-07$ \\
\hline 205064_at & SPRR1B & Small proline-rich protein 1B (cornifin) & 65.3558 & $6.94 \mathrm{E}-08$ \\
\hline 230835_at & KRTDAP & Keratinocyte differentiation-associated protein & 48.2006 & $7.42 \mathrm{E}-09$ \\
\hline 214370_at & S100A8 & S100 calcium binding protein A8 & 31.855 & $7.69 \mathrm{E}-09$ \\
\hline 33322_ì_at & SFN & Stratifin & 28.0939 & $1.02 \mathrm{E}-10$ \\
\hline 205916_at & S100A7 & S100 calcium binding protein A7 & 25.5473 & $8.06 \mathrm{E}-09$ \\
\hline 213796_at & SPRR1A & Small proline-rich protein 1A & 25.0762 & $1.68 \mathrm{E}-06$ \\
\hline 223278_at & GJB2 & Gap junction protein, beta $2,26 \mathrm{kDa}$ & 23.9597 & $3.74 \mathrm{E}-08$ \\
\hline 209351_at & KRT14 & Keratin 14 & 21.6562 & $3.32 \mathrm{E}-07$ \\
\hline 202917_s_at & S100A8 & S100 calcium binding protein A8 & 19.1306 & $1.68 \mathrm{E}-07$ \\
\hline 200606_at & $D S P$ & Desmoplakin & 15.9932 & $5.29 \mathrm{E}-07$ \\
\hline 205900_at & KRT1 & Keratin 1 & 15.8664 & $4.59 \mathrm{E}-06$ \\
\hline 1569410_at & FLG2 & Filaggrin family member 2 & 15.2786 & $6.81 \mathrm{E}-05$ \\
\hline 202286_s_at & TACSTD2 & Tumor-associated calcium signal transducer 2 & 13.9299 & $5.39 \mathrm{E}-06$ \\
\hline 204268_at & S100A2 & S100 calcium binding protein A2 & 13.2076 & $1.17 \mathrm{E}-07$ \\
\hline 201820_at & KRT5 & Keratin 5 & 12.569 & $3.79 \mathrm{E}-07$ \\
\hline 224328_s_at & $L C E 3 D$ & Late cornified envelope 3D & 12.4098 & $2.14 \mathrm{E}-08$ \\
\hline 207720_at & $L O R$ & Loricrin & 11.1667 & $2.42 \mathrm{E}-05$ \\
\hline 206032_at & DSC3 & Desmocollin 3 & 11.0151 & $3.82 \mathrm{E}-07$ \\
\hline 215704_at & FLG & Filaggrin & 9.96551 & $6.98 \mathrm{E}-05$ \\
\hline 236119_s_at & SPRR $2 G$ & Small proline-rich protein $2 \mathrm{G}$ & 9.65194 & $7.32 \mathrm{E}-09$ \\
\hline 39248_at & $A Q P 3$ & Aquaporin 3 (Gill blood group) & 9.5854 & $5.85 \mathrm{E}-05$ \\
\hline 204971_at & $\widetilde{C S T A}$ & Cystatin A (stefin A) & 8.72781 & $1.86 \mathrm{E}-05$ \\
\hline 214599_at & IVL & Involucrin & 8.57625 & $3.29 \mathrm{E}-07$ \\
\hline 207324_s_at & DSC1 & Desmocollin 1 & 8.29204 & $8.60 \mathrm{E}-06$ \\
\hline 209260_at & $S F N$ & Stratifin & 8.14597 & $1.64 \mathrm{E}-08$ \\
\hline 206642_at & DSG1 & Desmoglein 1 & 7.79091 & $3.18 \mathrm{E}-09$ \\
\hline 204855_at & SERPINB5 & Serpin peptidase inhibitor, clade B (ovalbumin), member 5 & 7.51157 & $3.00 \mathrm{E}-08$ \\
\hline 231771_at & GJB6 & Gap junction protein, beta $6,30 \mathrm{kDa}$ & 7.46408 & $3.68 \mathrm{E}-07$ \\
\hline 209720_s_at & SERPINB3 & Serpin peptidase inhibitor, clade B (ovalbumin), member 3 & 7.13291 & $7.10 \mathrm{E}-05$ \\
\hline 204475_at & $M M P 1$ & Matrix metallopeptidase 1 (interstitial collagenase) & 7.12341 & $8.74 \mathrm{E}-04$ \\
\hline 217528_at & CLCA2 & Chloride channel accessory 2 & 7.10544 & $2.12 \mathrm{E}-05$ \\
\hline 231211_s_at & $Y I F 1 B$ & Yip1 interacting factor homolog B (S. cerevisiae) & 7.08074 & $1.85 \mathrm{E}-04$ \\
\hline
\end{tabular}

Abbreviations: NCBI, National Center for Biotechnology Information; SLN, sentinel lymph node.

${ }^{\mathrm{a}}$ FLJ39739 /// LOC100286793 /// LOC728855 /// LOC728875 (Additional gene symbols), '—' uncharacterized genes. 


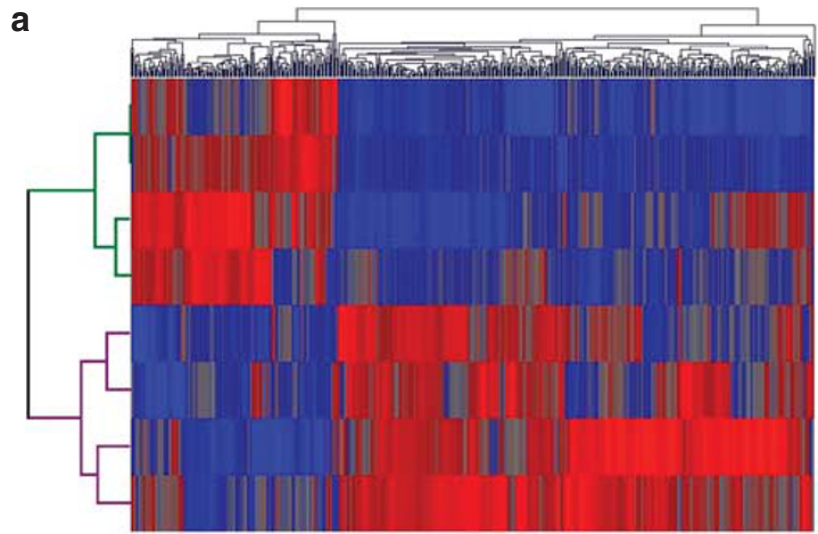

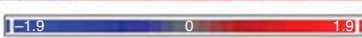

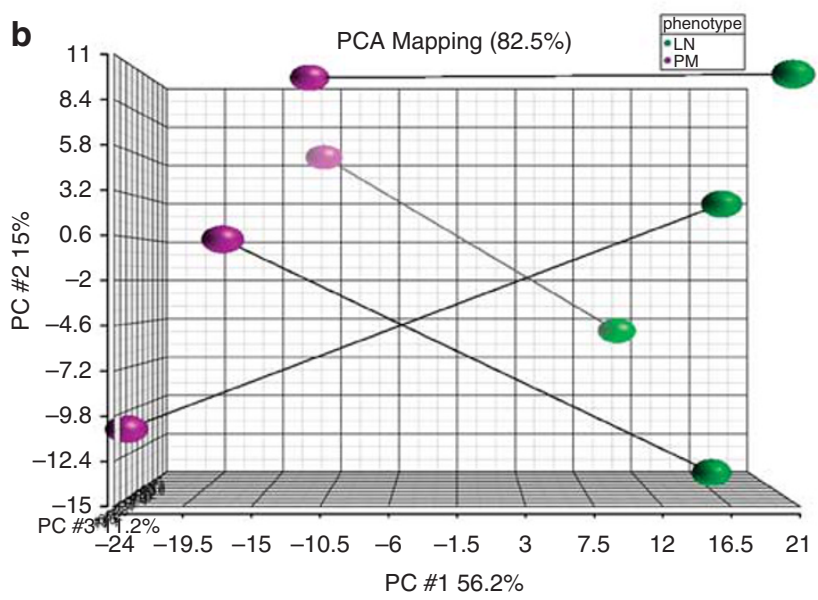

Figure 2 Analysis of primary melanomas vs sentinel lymph node melanoma metastases in paired samples. (a) Hierarchical clustering of eight samples resulted in two relatively distinct clusters of four sentinel lymph node melanoma metastases that had differential gene expression from the four paired primary melanomas. Each row represents each sample tested and each column represents a single probe. On the hierarchical tree at the left side of the diagram, the upper half (green) indicates the sentinel lymph node melanoma metastases and the lower half (purple) indicates the primary melanomas. Relative gene expression is color represented: red is higher-level expression, blue is lower-level expression, and grey is no change. (b) Principal component analysis of primary melanomas vs sentinel lymph node melanoma metastases in paired samples. Analysis resulted in two relatively distinct components of four sentinel lymph node melanoma metastases (green) and four primary melanomas (purple). Pairs from the same patient are denoted by lines.

products showed strong positive cytoplasmic staining in the tumor cells of sentinel lymph node metastases (Figure 3d and f, respectively). Melanoma cells at the primary site did not express either of these two markers (Figure 3c and e). Immunohistochemistry using antibodies to the other selected markers (MS4A1, SFN, KER6, SERPINB5, MMP1, and $S 100 A 8$ ) resulted in negative or equivocal staining of tumor cells without detectable differences in extent of epitope expression between the tumor cells of primary and metastatic tumors. Antibodies to gene products selected from the list of genes showing 'decreased expression' in the sentinel lymph node metastases list (Table 2b, SFN, KER6, SERPINB5, MMP1, and S100A8) stained epidermal keratinocytes and the epithelial cells of adnexal structures in the primary melanoma. The antibody to MS4A1 (also known as CD20) stained peritumoral and intratumoral B lymphocytes of the primary specimen, in addition to the native B-lymphocytes of the node specimen.

\section{Discussion}

The present investigation sought to identify genes implicated in the processes of lymphatic metastasis. The DNA-microarray gene expression profiling data clearly differentiated primary melanomas from sentinel node metastases. Hierarchical clustering illustrated distinct patterns of differentially expressed genes nearly identical in the analyses; autologous, non-autologous, and autologous and non-autologous samples combined. Data from the autologous paired samples (Figure 2) indicate that the differential gene expression profiles observed are due to specific biology-related altered transcription of selected genes by melanoma cells as they evolve, rather than to genetic heterogeneity between melanoma samples from different patients. There were 576 genes that had a statistically significant two-fold or greater difference in expression between primary and metastatic melanomas from the total sample analysis. For technical reasons (provision of sufficient tumor tissue for analysis), most of our cases were locally advanced (thick and deeply invasive), which limits the extent that our findings can be extrapolated to earlier, thinner primaries. It is certainly possible that the genes involved in lymphatic metastasis may be altered at an early stage of primary melanoma evolution. The multiple cumulative genetic changes identified in 'advanced' (thick and deeply invasive) primary melanomas by DNA microarray analysis may obscure the specific gene alterations that are essential for lymphatic invasion. Differences in gene expression between thin and thick primary melanomas have been reported. ${ }^{11}$

Among the 576 genes that distinguished primaries and metastases, most genes that were associated with metastases (402) showed decreased expression relative to the primary lesions. Previous gene expression studies of melanocytic tumors also reported that the predominant change was decreased expression of genes in advanced malignancy. ${ }^{14,15}$ Among genes that were decreased (Table 2b), SFN showed the greatest and most significant decrease. Stratifin is a protein that was initially identified in keratinizing stratified squamous epithelium and has a regulatory role in the G2/M cell cycle check point and cell death. Stratifin is also a tumor suppressor that is downregulated in cancers of breast, stomach, colon, lung, liver, pancreas, oral cavity, vulva, and urinary 

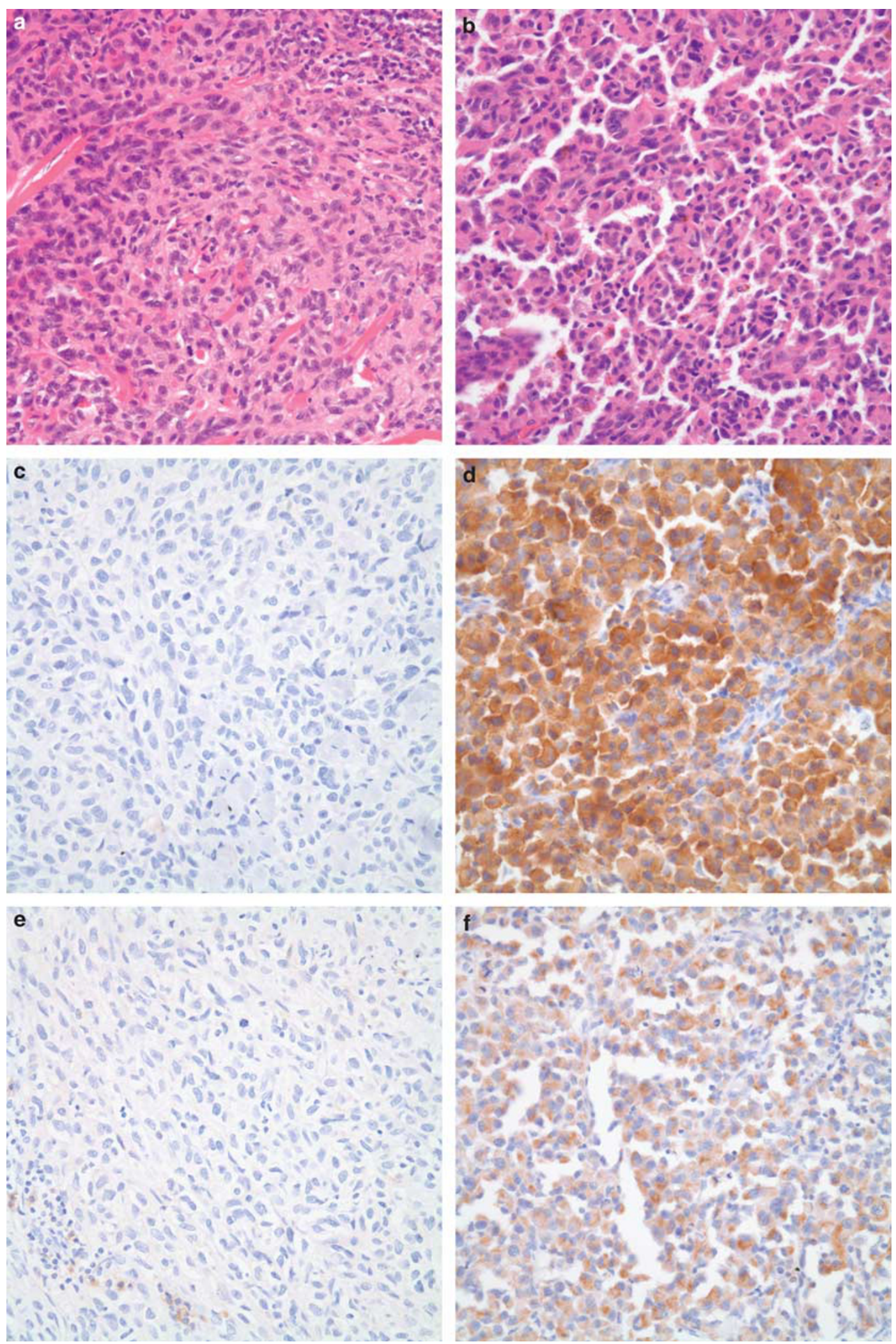

Figure 3 Validation with immunohistochemistry. Sections (a), (c), and (e) are primary melanoma samples. Sections (b), (d), and (f) are metastatic sentinel lymph node samples. Each were stained as follows: (a) and (b) with hematoxylin and eosin, (c) and (d) with MAGEC1 immunohistochemistry, (e) and (f) with FCRL1 immunohistochemistry. 
bladder. ${ }^{21}$ Prior studies have reported that decreased expression of stratifin was associated with metastasis in melanoma. ${ }^{14,15}$ SERPINB5, a tumor suppressor gene, with decreased expression associated with tumorigenesis has been implicated in development of cancers of the breast, prostate, thyroid, skin, and colon. ${ }^{22}$ Smith et $a l,{ }^{16}$ using the same Affymetrix Gene Chip Human U133 Plus 2.0. used in this study, have reported similar findings with SERPINB5. Although few of the genes identified in the downregulated set are consistent with the mechanism of metastasis, that is, decreased expression of tumor suppressor genes, there are multiple genes on the list that challenged our results. Within our data set are several genes previously characterized as keratinocyte-associated ( $L C E 2 B, S B S N, K R T 80, K R T D A P$, KRT6A, SPRR2G, SPRR1B, LCE3D, and KRT5). However, several prior studies of gene expression have reported downregulation of keratinocyte-related proteins, such as keratins, associated with melanoma. ${ }^{14-16}$ Others have encountered an increase in keratin genes in advanced melanoma. ${ }^{23}$ Unexpectedly high expression of keratin-associated proteins in primary/early stage melanomas relative to metastases has been a matter of considerable concern to previous investigators. Contamination of test specimens by keratinocytes from epidermis or skin appendages has been considered possible and our validation studies appear to support this possibility. Immunohistochemistry with selected 'downregulated' markers (SFN, KER6, SERPINB5, MMP1, and S100A8) showed staining of epithelial keratinocytes and adnexal structures in the primary melanomas. Keratinocyte contamination thus may partially explain at least some of the items on the list of 'downregulated' keratinocyte-related genes in Table 2a. We cannot, however, exclude the possibility that the list may also contain genes that are specifically relevant to melanoma, as keratins are widely distributed and function in determining basic attributes, such as cell shape and cell-cell adhesion. More extensive validation studies will be necessary to clarify whether melanoma-related keratin genes may co-exist with contaminant genes. It seems very likely that the microarray results for these markers were due to inclusion of (contaminating) epithelial cells or lymphocytes in the specimens that were submitted for RNA extraction. Preparation of a pure tumor cell preparation is clearly highly desirable, but technically demanding.

Although most differentially expressed genes were downregulated, there were numerous upregulated genes that correlated with sentinel lymph node metastasis. FCRL1 is a transmembrane glycoprotein, previously reported to be expressed in melanocytes and melanoma cells. ${ }^{24}$ This gene is also expressed in B-cells and has been used in immunotherapy of B-cell leukemias/lymphomas. ${ }^{25}$ Inozume et $a l^{24}$ reported that FCRL1 is immunogenic in melanoma, and that there are antibodies to FCRL1 in serum from melanoma patients, suggesting that FCRL1 may have a potential use in immunotherapy targeted to prevention of lymphatic metastasis of melanoma. Some of the upregulated gene products are oncogenes or potential tumor promoters. MAGEC1 and other subtypes of the $M A G E$ I subgroup are generally expressed at higher levels in tumor cells and germ cells, relative to normal mature somatic cells. This family of proteins is increased in melanomas, gastrointestinal carcinomas, esophageal carcinomas, and pulmonary carcinomas. ${ }^{26}$ There is clear evidence of increased MAGEC1 in metastatic melanoma in our study, and by analogy with FCRL1, this raises the possibility of a role for MAGEC1 in the mechanisms of lymphatic metastasis. Results of immunohistochemical studies of gene products for MAGEC1 and FCRL1 correlated with the microarray results, which showed significantly increased protein expression of MAGEC1 and FCRL1 in the samples from sentinel lymph node metastases.

Functional analysis indicates that the genes that were downregulated in metastases in our study are involved in cell cycle regulation, cell adhesion, protease inhibitory activity, and keratinocyte-associated functions. Some of these genes have been previously reported to be involved in tumorigenesis in melanoma. Loss of cell cycle regulation and cell adhesion is associated with metastasis. Our observation that these classes of genes are 'downregulated' in association with sentinel lymph node metastasis is thus in line with prior studies and current concepts of tumor biology. Additionally, some of the 'upregulated' genes associated with sentinel lymph node metastases were oncogenes or tumor promoters, findings that are also in line with current knowledge of tumor biology.

In summary, DNA microarray expression profiling distinguishes primary melanoma from melanoma metastatic to the sentinel node on the basis of distinct differential expression of multiple genes. Some of these genes are well characterized and confirm the results of previously published studies. Some of the genes that appeared differentially expressed relate to non-melanoma tissues that are co-located with melanoma cells in the skin and lymph nodes. The presence of these 'contaminant' genes requires very meticulous tumor sampling and the development of approaches that will identify and separate contaminant genes from genes that are truly melanoma-related. There remain substantial variations in the findings reported by different laboratories. These may be explained by evaluation of different metastases (nodal vs visceral), the use of different microarray gene chip technology, the type of melanoma specimens analyzed (cell cultures vs fresh frozen melanoma $v s$ formalin-fixed, paraffinembedded tissue), degree of cellular purity of the study material, and mRNA purification techniques. The heterogeneous and complex nature of melanoma may also contribute to the inter-investigator discrepancies observed. ${ }^{23}$ Despite inter-observer variations, it is clear that there are substantial 
differences in gene expression between melanomas at different stages of progression, in this study, between primary melanomas and melanoma metastases in sentinel nodes.

\section{Acknowledgements}

This research is supported by the UCLA Department of Pathology and Laboratory Medicine Translation Research Fund (SWB, SSK: Grant number 401450XV-62220) and the NIH 2-R01-CA-29605 (AJC and $\mathrm{RRH}$ ), administered as a subcontract from The John Wayne Cancer Institute, PI, DL Morton, MD. We thank Jonathan Braun, MD, PhD, and Wayne Grody, $\mathrm{MD}, \mathrm{PhD}$, for their review and constructive criticisms of the manuscript.

\section{Disclosure/conflict of interest}

The authors declare no conflict of interest.

\section{References}

1 Balch CM, Gershenwald JE, Soong S-J, et al. Final version of 2009 AJCC melanoma staging and classification. J Clin Oncol 2009;27:6199-6206.

2 Morton DL, Thompson JF, Cochran AJ, et al. SentinelNode Biopsy or Nodal Observation in Melanoma. N Engl J Med 2006;355:1307-1317.

3 Liphshutz RJ, Morris D, Chee M, et al. Using oligonucleotide probe arrays to access genetic diversity. Biotechniques 1995;19:442-447.

4 Schena M, Shalon D, Davis RW, et al. Quantitative monitoring of gene expression patterns with a complementary DNA microarray. Science 1995;270: 467-470.

5 Quackenbush J. Microarray analysis and tumor classification. N Engl J Med 2006;354:2463-2472.

6 Bittner M, Meltzner P, Chen Y, et al. Molecular classification of cutaneous malignant melanoma by gene expression profiling. Nature 2000;406:536-540.

7 Koh SS, Opel ML, Wei J-P, et al. Molecular classification of melanomas and nevi using gene expression microarray signatures and formalin-fixed and paraffinembedded tissue. Mod Pathol 2009;22:538-546.

8 Seykora JT, Jih D, Elenitsas R, et al. Gene expression profiling of melanocytic lesions. Am J Dermatopathol 2003;25:6-11.

9 Talantov D, Mazumder A, Yu JX, et al. Novel genes associated with malignant melanoma but not benign melanocytic lesions. Clin Cancer Res 2005;11: 7234-7242.

10 Nambiar S, Mirmohammadsadegh A, Doroudi R, et al. Signaling networks in cutaneous melanoma metastasis identified by complementary DNA microarrays. Arch Dermatol 2005;141:165-173.
11 Winnepenninckx V, Lazar V, Michiels S, et al. Gene expression profiling of primary cutaneous melanoma and clinical outcome. J Natl Cancer Inst 2006;98: 472-482.

12 Haqq C, Nosrati M, Sudilovsky D, et al. The gene expression signatures of melanoma progression. Proc Natl Acad Sci USA 2005;102:6092-6097.

13 Jensen EH, Lewis JM, McLoughlin JM, et al. Downregulation of pro-apoptotic genes is an early event in the progression of malignant melanoma. Ann Surg Oncol 2007;14:1416-1423.

14 Riker AI, Enkemann SA, Fodstad O, et al. The gene expression profiles of primary and metastatic melanoma yields a transition point of tumor progression and metastasis. BMC Med Genomics 2008;1:1-16.

15 Jaeger J, Koczan D, Thiesen H-J, et al. Gene expression signatures for tumor progression, tumor subtype, and tumor thickness in laser-microdissected melanoma tissues. Clin Cancer Res 2007;13:806-815.

16 Smith AP, Hoek K, Becker D. Whole-genome expression profiling of the melanoma progression pathway reveals marked molecular differences between nevi/ melanoma in situ and advanced-stage melanomas. Cancer Biol Ther 2005;4:1018-1029.

17 Mandruzzato S, Callegaro A, Turcatel G, et al. A gene expression signature associated with survival in metastatic melanoma. J Transl Med 2006;4:1-11.

18 Hoek K, Rimm DL, Williams KR, et al. Expression profiling reveals novel pathways in the transformation of melanocytes to melanomas. Cancer Res 2004;64: 5270-5282.

19 Conway C, Mitra A, Jewell R, et al. Gene expression profiling of paraffin-embedded primary melanoma using the DASL assay identifies increased osteopontin expression as predictive of reduced relapse-free survival. Clin Cancer Res 2009;15:6939-6946.

20 Rangel J, Nostrati M, Torabian S, et al. Osteopontin as a molecular prognostic marker for melanoma. Cancer 2008;112:144-150.

21 Moreira JM, Gromov P, Celis JE. Expression of the tumor suppressor protein $14-3-3 \sigma$ is down-regulated in invasive transitional cell carcinomas of the urinary bladder undergoing epithelia-to-mesenchymal transition. Mol Cell Proteomics 2004;3:410-419.

22 Khalkhali-Ellis Z. Maspin: the new frontier. Clin Cancer Res 2006;12:7279-7283.

23 Hoek KS. Melanoma progression, gene expression and DNA microarrays. G Ital Dermatol Venereol 2009; 144:1-11.

24 Inozume T, Matsuzaki Y, Kurihara S, et al. Novel melanoma antigen, FCRL/FREB, identified by cDNA profile comparison using DNA chip Are immunogenic in multiple melanoma patients. Int J Cancer 2005;114: 283-290.

$25 \mathrm{Du} \mathrm{X}$, Nagata $\mathrm{S}$, Ise $\mathrm{T}$, et al. FCRL1 on chronic lymphocytic leukemia, hairy cell leukemia, and B-cell non-Hodgkin lymphoma as target of immunotoxins. Blood 2008;111:338-343.

26 Xiao J, Chen HS. Biological functions of melanomaassociated antigens. World J Gastroenterol 2004;10: 1849-1853. 